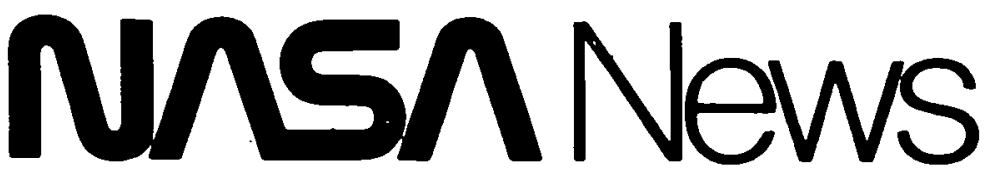

Natıonal Aeronaulics and Space Admınistration

Washington, D.C. 20546 AC 202 755.8370

For Release

Nicholas Panagakos

Headquarters, Washington, D.C.

IMMEDIATE

(Phone: 202/755-3680)

Don Bane

NASA Jet Propulsion Laboratory, Pasadena, Calif.

(Phone: 213/354-5011)

$\stackrel{1}{0}$

RELEASE NO: 77-177

\title{
INVESTIGATORS NAMED FOR JUPITER ORBITER PROBE
}

One hundred and fourteen scientists have been tentatively chosen by NASA to participate in the Jupiter-Orbiter-withProbe (JOP) mission scheduled for 1981-82.

The team was selected from more than 500 scientists who submitted proposals for investigations in response to NASA's invitation a year ago.

Scheduled to become the first planetary spacecraft to be carried aboard NASA's Space Shuttle, JOP is designed to conduct the most detailed scientific investigation yet of Jupiter, its environment and moons, including the first direct measurements of the planet's atmosphere. 
The mission is composed of an orbiter which will circle the planet for at least 20 months and a probe which will plunge deeply into Jupiter's atmosphere.

Jupiter, the largest planet, contains more matter than all the other planets combined and it holds many clues to the origin and evolution of the solar system. To understand the solar system, scientists need to understand Jupiter: to probe its atmosphere, to map its magnetic field and radiation belts and to examine the huge satellites that orbit it.

Although previous spacecraft have flown or will fly past Jupiter, JOP offers the first opportunity to make direct as well as remote measurements of the giant planet, its environment and its satelites from various orbiting positions over a long period of time.

The basic mission as now envisioned will involve probe separation from the orbiter while the two spacecraft are approximately 55 days away from the planet. The two spacecraft will continue on separate flight paths until the probe enters the Jovian atmosphere and relays its data back to Earth via the orbiter. Approximately 30 minutes of data will be transmitted to the orbiter during the high speed descent. 
After termination of the entry probe phase, the orbiter will be inserted into its initial Jovian orbit by an onboard retro propulsion system. Subsequent propulsion adjustments will permit the spacecraft to fly close to the Jovian moon Ganymede and to make more distant encounters with the other large Galilean satellites of Jupiter.

A single launch by NASA's Space Shuttle is planned for late 1981 or early 1982 and flight time to Jupiter will be just under three years.

of the 114 scientific investigators who will make up the JOP team, 13 will have interdisciplinary tasks. They will aid in mission planning and in coordinating science investigations to assure the best overall mission results. The others will be assigned to 17 individual experiment areas. Principal Investigators in three of these areas are from foreign countries.

NASA's Office of Space Science has assigned management of the JOP project to NASA's Jet Propulsion Laboratory, Pasadena, Calif. NASA's Ames Research Center, Mountain View, Calif., will manage the probe system. The NASA Space Transportation System (Space Shuttle and Interim Upper Stage) will be managed by Johnson Space Center, Houston, Tex. 
The instruments and the Principal Investigators for the orbiter portion of JOP are:

- Imaging system with a 1.5-meter (4.9-foot) objective lens. For the first time aboard a spacecraft, it makes use of solid state charge-coupled devices (CCD) to form the images. The CCD gives broader spectral response and better resolution than previous spacecraft cameras. Dr. M.J.S. Belton of Kitt Peak National Observatory will be the Imaging Team Leader.

- Near-infrared mapping spectrometer. The instrument will make images in many different wavelength ranges. It will allow identification of materials on the surfaces of Jupiter's satellites. Dr. T.V. Johnson of Jet Propulsion Laboratory will be Principal Investigator.

- Ultraviolet spectrometer. The instrument will study the dense atmosphere of Jupiter and the rare, tenuous atmospheres of its satellites. Dr. C.W. Hord of University of Colorado will be Principal Investigator.

- Photopolarimeter-radiometer. The instrument will measure temperatures at various heights in Jupiter's atmosphere and on the satellite's surfaces. Dr. A.A. Lacis of NASA's Goddard Institute for Space Studies will be Principal Investigator. 
- Magnetometer. The instrument will measure magnetic fields and their variations in the vicinity of Jupiter and its satellites. It will measure variations caused by the satellites as they orbit Jupiter. Dr. Margaret Kivelson of UCLA will be Principal Investigator.

- Plasma. The instrument studies low-energy particles in the Jovian magnetosphere and interplanetary space. Dr. L.A. Frank of University of Iowa will be Principal Investigator.

- Energetic particles. The instrument will measure high-energy particles trapped by Jupiter's large, intense magnetic field -- similar to the Van Allen radiation belts that surround Earth. Dr. D.J. Williams of National Oceanic and Atmospheric Administration (NOAA) will be Principal Investigator.

- Electron emitter. The instrument studies static charges built up on the spacecraft as it passes through high-energy regions around Jupiter. It will attempt to control the charges so that engineering systems and science instruments are not damaged by possible "arcing" between areas of different charge. Dr. R. Grard of ESTEC, The Netherlands, will be Principal Investigator. 
- Plasma wave. The instrument studies how plasma waves and changes with time; the changes are believed to be caused by magnetic and electric fields near Jupiter and in interplanetary space. Dr. D.A. Gurnett of University of Iowa will be Principal Investigator.

- Dust measurement. The instrument will measure size, speed and electron charge of small particles (like meteorites) near Jupiter and its satellites. Dr. E. Grun of the Max Planck Institute, Federal Republic of Germany, will be Principal Investigator.

- Radio science and celestial mechanics. The experiment uses spacecraft radio signals to measure the path of the spacecraft, the density and depth of the atmosphere and any gravity anomalies that might be caused by mass concentrations in Jupiter or its satellites.

Instruments on the probe which will make direct measurements as it descends through the atmosphere of Jupiter, and Principal Investigators are:

- Helium interferometer. The instrument will measure with extreme accuracy the ratio of hydrogen to helium in the atmosphere. 
The measurement is of significance to cosmology, since it helps scientists understand whether the universe is "closed" as the bit-band theory says, or open, as other theories say. Dr. Ulf von Zahn of University of Bonn, Federal Republic of Germany, will be Principal Investigator.

- Mass spectrometer. The instrument works in cooperation with the helium interferometer. The spectrometer will analyze the composition of the Jovian atmosphere. Dr. H.B. Niemann of NASA's Goddard Space Flight Center will be Principal Investigator.

- Atmospheric structure. By measuring deceleration of the probe as it passes tl rough the atmosphere, scientists can measurfatmospheric pressure and temperatures as they change with altitude. Dr. Alvin Sieff of Ames Research Center will be Principal Investigator.

- Nephelometer. The instrument studies clouds, particle sizes and locations in the atmosphere. Dr. Boris Ragent of Ames Research Center will be Principal Investigator. 
$-8-$

- Net-flux radiometer. The instrument will measure energy radiated and received by Jupiter. (Jupiter appears to radiate 2.5 times the energy that it receives from the Sun.) Dr. R.W. Bose of Ames's Research Center will be Principal Investigator.

- Sferics receiver. The instrument will measure radio $\therefore$ static generated by lightning discharges in Jupiter's atmosphere, and will also be able to detect the light given off by lightning flashes. Dr. L.J. Lanzerotti nf Bell Laboratories will be Principal Investigator.

-end- 